\title{
Il ruolo della renina plasmatica nella titolazione della terapia sostitutiva con mineralcorticoidi in pazienti affetti da insufficienza surrenalica primaria
}

\author{
Riccardo Pof ${ }^{1}$. Elisa Giannetta ${ }^{1}$
}

Accettato: 5 gennaio 2021 / Pubblicato online: 9 agosto 2021

(c) The Author(s) 2021

Sommario L'ottimizzazione della terapia con mineralcorticoidi in pazienti con insufficienza surrenalica primaria non ha ancora trovato consenso universale. Questa rassegna riporta i dati di una vasta coorte di pazienti affetti da insufficienza surrenalica primaria con l'obiettivo di esplorare la relazione tra la dose sostitutiva dei mineralcorticoidi (MC), la concentrazione plasmatica di renina (CPR) e variabili cliniche ambulatoriali (elettroliti, pressione sanguigna, PA, e parametri antropometrici) al fine di identificare marcatori utili per guidare la titolazione della dose MC. I risultati hanno mostrato un' estrema variabilità nei valori di CPR e nella dose di MC. Nell' analisi univariata, la dose di MC era direttamente proporzionale alla CPR ma non correlava con i livelli di PA. Utilizzando modelli di regressione multipla, il sodio era l'unica variabile utile a predire la CPR. Nell'analisi longitudinale, la variazione della dose di MC era correlata alla variazione dei livelli sierici di potassio ma non alla PA o alla CPR. In conclusione, la relazione tra la dose di MC e la CPR è complessa e, pertanto, la titolazione dei MC non dovrebbe essere basata solo sulla normalizzazione della CPR, ma anche su parametri clinici come la PA e la concentrazione di elettroliti.

Parole chiave Renina - Mineralcorticoidi · Insufficienza surrenalica primaria

\section{Proposto da Elisa Giannetta.}

Informazioni Supplementari La versione online contiene materiale supplementare disponibile su

https://doi.org/10.1007/s40619-021-00927-z.

$\triangle$ R. Pofi

riccardo.pofi@uniroma1.it

1 Dipartimento di Medicina Sperimentale, Sezione di Fisiopatologia Medica, Sapienza Università di Roma, Roma, Italia

\section{Introduzione}

L'insufficienza surrenalica primaria (ISP) è una condizione determinata da malattie che coinvolgono direttamente la corteccia surrenalica e, se non riconosciuta o inappropriatamente trattata, è potenzialmente fatale. Lo spettro clinico è caratterizzato da scarsa produzione o azione dei glucocorticoidi (GC), con o senza concomitante deficit di mineralcorticoidi (MC) e androgeni surrenalici. Nella maggior parte dei casi, l'ISP è causata da un'adrenalite autoimmune (malattia di Addison). L'iperplasia surrenalica congenita (ISC), invece, è una forma diversa di ISP, causata da un gruppo di rare malattie autosomiche recessive derivanti da mutazioni nei geni che codificano per enzimi critici per la biosintesi degli steroidi surrenalici. La forma più comune è causata da mutazioni nel Gene CYP21A2, responsabile della 21-idrossilazione, ed è causa di circa il $95 \%$ dei casi di ISC. L'alterata 21-idrossilazione può portare alla riduzione della sintesi di GC e MC. In particolare, la forma di ISC con perdita di sali (ISC-PS) è caratterizzata carenza sia di GC che MC. Nella ISC-PS e nella ISP, la terapia ormonale sostitutiva con GC che MC è essenziale per prevenire crisi surrenaliche potenzialmente letali.

Molta attenzione si è concentrata principalmente sulla corretta titolazione della terapia sostitutiva con GC in pazienti affetti da insufficienza surrenalica. Al contrario, sebbene siano trascorsi quasi 70 anni dall'introduzione della terapia sostitutiva con $\mathrm{MC}$ per i pazienti con ISC, finora le strategie di trattamento con MC e il loro monitoraggio sono stati valutati in un numero limitato di studi e, ad oggi, esistono pochi dati utili a ottimizzare la dose di MC. Ad oggi non sono stati individuati, infatti, marcatori che siano risultati affidabili per il monitoraggio della terapia. Una terapia sostitutiva con $\mathrm{MC}$ che sia personalizzata e accuratamente titolata è di cruciale importanza in questi pazienti, al 
Tabella 1 Caratteristiche basali di 188 pazienti con insufficienza surrenalica. I dati sono espressi come mediana (intervallo). $I S C$ - $P S$, iperplasia surrenalica congenita con perdita di sali; $M C$, mineralcorticoidi; $P A M$, pressione arteriosa media; $C P R$, concentrazione plasmatica di renina; $B M I$, indice di massa corporea

\begin{tabular}{llll}
\hline & Intera coorte & ISC-PS & M. Addison \\
\hline $\mathrm{n}$ & 188 & 149 & 39 \\
Valutazioni (n) & 386 & 347 & 39 \\
Età $(\mathrm{anni})$ & $27(16-84)$ & $25(16-67)$ & $49(17-84)$ \\
$\mathrm{BMI}\left(\mathrm{kg} / \mathrm{m}^{2}\right)$ & $29(15-50)$ & $29(15-50)$ & $26(17-33)$ \\
$\mathrm{Na}^{+}(\mathrm{mmol} / \mathrm{L})$ & $140(126-146)$ & $140(130-146)$ & $138(126-143)$ \\
$\mathrm{K}^{+}(\mathrm{mmol} / \mathrm{L})$ & $4,2(2,7-5,9)$ & $4,2(2,7-5,9)$ & $3,9(3,1-4,7)$ \\
$\mathrm{MAP}(\mathrm{mmHg})$ & $93(70-125)$ & $93(70-125)$ & $93(75-120)$ \\
Dose giornaliera MC $(\mu \mathrm{g} /$ day $)$ & $150(25-400)$ & $150(25-400)$ & $100(50-300)$ \\
$\mathrm{CPR}(\mathrm{mUI} / \mathrm{mL})$ & $86(0,6-3166)$ & $87(0,6-3166)$ & $82(4,2-2879)$ \\
\hline
\end{tabular}

fine di migliorare la qualità di vita e ridurre le complicanze cardiovascolari a lungo termine.

Le più recenti linee guida della Endocrine Society [1] sulla gestione clinica della ISC suggeriscono una terapia sostitutiva a base di fludrocortisone da 50 a $200 \mu \mathrm{g} /$ die.

Negli adulti, la dose di MC (e/o l'integrazione di sale) dovrebbe essere titolata clinicamente tenendo in considerazione la pressione arteriosa (PA), valutando il desiderio di cibi salati e la presenza di edemi periferici. Tuttavia, questi sintomi e segni non sono sempre affidabili e, pertanto, sono necessari marcatori più obiettivi. Tra questi, sebbene manchino prove convincenti a sostegno, è suggerita la misurazione dei livelli sierici di sodio $\left(\mathrm{Na}^{+}\right)$, potassio $\left(\mathrm{K}^{+}\right)$e della Concentrazione Plasmatica della Renina (CPR), che possono essere utili nel guidare il clinico nella titolazione della dose sostitutiva di MC.

Le linee guida [1] suggeriscono, inoltre, che la sostituzione con $\mathrm{MC}$ dovrebbe mirare a raggiungere normali livelli di pressione arteriosa, di potassio sierico, mantenendo la CPR all'interno del limite superiore dell'intervallo di riferimento.

Tuttavia, la complessità dei sistemi fisiologici di regolazione della CPR, nonché della sua estrema variabilità in risposta a una molteplicità di fattori, rende difficile utilizzarla per la titolazione della terapia sostitutiva con MC, che spesso rappresenta una vera e propria battaglia per il clinico che mira a ottenere un buon controllo di malattia e una qualità di vita adeguata. In passato, Oelkers e colleghi hanno dimostrato che, quando titolata al limite superiore dell'intervallo di riferimento, l'attività della renina plasmatica era più strettamente correlata alla dose di MC rispetto ai soli livelli di $\mathrm{Na}^{+}$e $\mathrm{K}^{+}$[2]. Al contrario, lo studio di Thompson ha successivamente mostrato che l'attività della renina plasmatica non era un marcatore in grado di distinguere un'adeguata terapia sostitutiva da una terapia eccessiva [3], sollevando quindi dubbi sull'affidabilità della CPR nell'ottimizzazione della dose di MC.

In tale contesto, l'obiettivo del nostro studio è stato quello di esplorare eventuali relazioni intercorrenti tra la dose di MC assunta dal paziente e le variabili cliniche e biochimiche raccolte nella pratica clinica di tutti i giorni, al fine di determinare se possano essere utili per guidare il clinico verso la titolazione della dose di MC appropriata.

\section{Metodologia di analisi e risultati}

Nello studio recentemente pubblicato, abbiamo pertanto effettuato un'analisi retrospettiva dei dati presenti nel registro internazionale delle ISC (www.icah.org) raccolti dal 1982 al 2018 in 14 centri in 7 paesi (Regno Unito, Brasile, Italia, Turchia, Israele, Bulgaria e Germania), unitamente a database locali contenenti pazienti affetti da ISP e ISC in trattamento sostitutivo con MC [4].

In totale sono stati inclusi dati clinici e biochimici derivanti da 386 valutazioni su 188 pazienti: 149 affetti da ISC-PS e 39 da Malattia di Addison. È stata inoltre eseguita un'analisi longitudinale in 112 pazienti di cui si disponeva di visite consecutive. I dati demografici dei pazienti sono presentati nella Tabella 1 .

Sei variabili sono state prese in considerazione nel modello di analisi multivariata finale: il sodio sierico $\left(\mathrm{Na}^{+}\right)$, il potassio sierico $\left(\mathrm{K}^{+}\right)$, la pressione arteriosa media (PAM), la CPR, la dose giornaliera sostitutiva di MC assunta dal paziente, l'età e l'indice di massa corporea (BMI). Tutte le variabili che risultavano significative nel modello iniziale sono state, quindi, testate come variabili dipendenti nelle successive analisi di regressione multipla.

Per quanto riguarda i pazienti affetti da ISP-PS, il 17$\mathrm{OH}$ progesterone (17-OHP) e l'androstenedione sono stati considerati nell'analisi univariata basale come parametri indiretti di aderenza alla terapia sostitutiva con GC.

I pazienti sono stati inoltre stratificati per il livello di renina basale (CPR bassa, normale e alta in base all'intervallo di riferimento locale) o, nell'analisi longitudinale, per la variazione della dose di MC (dose invariata, ridotta e aumentata).

L'analisi basale ha mostrato una grande variabilità sia nella dose giornaliera di MC assunta dal paziente che nella CPR, con valori che variavano da 0,6 a $3166 \mu \mathrm{IU} / \mathrm{mL}$ 
Tabella 2 Modello di regressione multipla. Il $p$-value deve essere interpretato con la correzione di Bonferroni, se significativo è evidenziato in grassetto e con asterisco

\begin{tabular}{|c|c|c|c|c|}
\hline \multirow{2}{*}{$\frac{\text { Modello } 1\left(p<0,001^{*}\right)}{\text { Variabili indipendenti }}$} & \multicolumn{4}{|c|}{ Variabile dipendente: CPR } \\
\hline & $\mathrm{B}$ & $95 \%$ CI limite inferiore & $95 \%$ CI limite superiore & $p$ \\
\hline Dose giornaliera $\mathrm{MC}$ & 0,001 & $-0,001$ & 0,002 & 0,515 \\
\hline $\mathrm{K}^{+}$ & 0,044 & $-0,201$ & 0,289 & 0,723 \\
\hline $\mathrm{Na}^{+}$ & $-0,091$ & $-0,126$ & $-0,055$ & $<0,001 *$ \\
\hline MAP & 0,005 & $-0,006$ & 0,016 & 0,351 \\
\hline Age & $-0,006$ & $-0,013$ & 0,001 & 0,072 \\
\hline BMI & 0,004 & $-0,012$ & 0,020 & 0,591 \\
\hline Modello $2(p=0.017 *)$ & \multicolumn{4}{|c|}{ Variabile dipendente: dose giornaliera MC } \\
\hline Variabili indipendenti & $\mathrm{B}$ & 95\% CI limite inferiore & 95\% CI limite superiore & $p$ \\
\hline $\mathrm{K}^{+}$ & $-17,523$ & $-43,350$ & 8,303 & 0,182 \\
\hline $\mathrm{Na}^{+}$ & 1,026 & $-3,072$ & 5,123 & 0,621 \\
\hline PRC & 5,846 & $-11,873$ & 23,565 & 0,515 \\
\hline MAP & 0,566 & $-0,593$ & 1,726 & 0,336 \\
\hline Age & 0,030 & $-0,722$ & 0,781 & 0,938 \\
\hline BMI & 2,812 & 1,183 & 4,441 & $\mathbf{0 , 0 0 1} *$ \\
\hline
\end{tabular}

(mediana $86 \mu \mathrm{IU} / \mathrm{mL}$ ). Quando stratificati in base agli intervalli di riferimento locali, l'8, il 31 e il $61 \%$ dei pazienti presentavano valori di PRC rispettivamente bassi, normali e alti.

L'analisi univariata ha dimostrato una correlazione positiva tra la dose giornaliera di MC e il BMI ( $\mathrm{r}=0,233 ; p<$ $0,001)$, l'età $(\mathrm{r}=0,116 ; p=0,023)$ e la CPR ( $\mathrm{r}=0,135$; $p=0,051)$, mentre non vi era alcuna relazione con i livelli sierici di $\mathrm{Na}^{+}$e $\mathrm{K}^{+}$o con la PAM. Considerando gli intervalli di riferimento locali, rispetto a coloro che presentavano valori di CPR ridotti, i pazienti con CPR elevata presentavano concentrazioni sieriche $\mathrm{di} \mathrm{Na}^{+}$più basse e concentrazioni di $\mathrm{K}^{+}$più elevate, mentre non si riscontravano correlazioni tra la dose giornaliera di MC, la CPR, il 17-OHP e l'androstenedione.

Al fine di determinare se una CPR elevata derivasse da una dose insufficiente o da una scarsa aderenza alla terapia con MC e GC, sono stati confrontati i livelli di 17-OHP e androstenedione tra i pazienti con CPR bassa, normale o alto: le analisi non hanno mostrato differenze significative.

L'analisi derivante dal modello di regressione multipla a 6 variabili ha mostrato che il $\mathrm{Na}^{+}$era l'unica variabile (debolmente) correlata alla CPR $(\mathrm{B}=-0,091 ; p<0,001)$ e che la dose giornaliera di MC era direttamente correlata al BMI ( $\mathrm{B}=2,812 ; p=0,001)$, ma non alla MAP o alla CPR.

Per quanto riguarda l'analisi longitudinale, al follow-up la dose giornaliera di MC è rimasta invariata nel $67 \%$ dei pazienti (gruppo 1), mentre nel 6\% (gruppo 2) è stata ridotta e nel 19\% (gruppo 3) aumentata. Non ci sono state variazioni significative della CPR all'interno dei singoli gruppi e le differenze tra i cambiamenti della CPR non erano diverse tra i 3 gruppi. Il modello di regressione multipla ha dimostrato che solo la concentrazione del sodio nella visita di controllo era associata alla variazione della $\mathrm{CPR}(\mathrm{B}=59,465 ; p$ $<0,001)$; tuttavia, non c'erano relazioni tra la variazione di CPR e della PAM, del K+ o della dose giornaliera di MC al follow-up. Come atteso, la variazione della dose giornaliera di MC era inversamente associata alla variazione di potassio $(\mathrm{B}=-3,104 ; p=0,002)$. Nessuna relazione, invece, è stata trovata tra la variazione di dose giornaliera di MC e la variazione di CPR, Na+ o PAM (Tabella 2).

\section{Discussione}

Nei pazienti con insufficienza surrenalica molta attenzione si è focalizzata sull'ottimizzazione della terapia sostitutiva con GC; tuttavia, può essere difficile distinguere clinicamente un'inadeguata supplementazione di GC e MC. È importante evitare un sovradosaggio del trattamento con i GC che, come è noto, è associato a significativi effetti avversi. Tenendo presente una possibile attività mineralcorticoidea dei GC comunemente usati (idrocortisone e prednisolone), è verosimile che la necessità di un aumento delle dosi sostitutive di GC sia in realtà secondario a una relativa carenza di MC piuttosto che di GC, sottolineando quindi la possibilità che molti pazienti con ISP siano inadeguatamente sostituiti con terapia con MC.

Questo potrebbe essere un importante fattore che contribuisce alla mancanza di relazione tra dose MC e CPR che abbiamo osservato in basale nel nostro studio. Nella nostra coorte, infatti, la CPR era debolmente correlata alle concentrazioni sieriche di $\mathrm{Na}^{+}$, ma non aveva alcuna relazione con le altre variabili cliniche (compresa la PAM) o, soprattutto, 
con la dose giornaliera sostitutiva di MC. Inoltre, l'analisi longitudinale suggerisce che i cambiamenti della dose di MC non erano associati a variazioni di CPR al follow-up. $\mathrm{Al}$ contrario, gli elettroliti sierici (in particolare il $\mathrm{K}^{+}$) sono strettamente e fortemente correlati alla dose $\mathrm{MC}$ sia al basale che nell'analisi longitudinale. Queste osservazioni potrebbero riflettere la normale fisiologia di controllo elettrolitico da parte del sistema renina-angiotensina-aldosterone, dato che i pazienti che mostravano livelli di PRC più elevati, avevano livelli di $\mathrm{Na}^{+}$più bassi (con il $\mathrm{Na}^{+}$che era anche l'unica variabile associata a cambiamenti della CPR nell'analisi longitudinale). Questo potrebbe suggerire una dose insufficiente della terapia sostitutiva con MC e una conseguente perdita di $\mathrm{Na}^{+}$, sebbene dobbiamo sottolineare che le analisi non sono state controllate per la dose di GC assunta dal paziente e, quindi, il contributo relativo dei GC sul bilancio del $\mathrm{Na}^{+}$ non è stato valutato.

Parallelamente, come previsto, l'associazione tra dose giornaliera di $\mathrm{MC}$ e i livelli sierici $\mathrm{di}^{+}$ha suggerito che dosi MC più elevate erano associate a concentrazioni sieriche $\mathrm{K}^{+}$inferiori.

Gli autori non vogliono mettere in dubbio l'utilità delle misurazioni della CPR e dell'aldosterone nella diagnosi di un deficit di secrezione di MC. Tuttavia, sfide significative sorgono quando questi parametri vengono utilizzati per l'aggiustamento della dose sostitutiva con MC. In tale contesto, una delle problematiche riguarda la difficoltà nella raccolta e gestione dei campioni ematici per l'analisi della CPR; inoltre, non esiste un intervallo di riferimento standard per la CPR accettato a livello internazionale e l'interpretazione dei risultati dipende essenzialmente da intervalli di riferimento stabiliti localmente. Inoltre, ci sono molti fattori che al momento del prelievo influenzano la CPR, tra cui il volume ematico, l'assunzione di sale, la gravidanza, la postura, la temperatura ambientale ed eventuali farmaci antiipertensivi e antinfiammatori non steroidei assunti dal paziente. Nella maggior parte dei centri, i campioni prelevati per la misurazione della CPR non sono standardizzati rispetto alla postura o alla tempistica di prelievo rispetto all'ultima dose di MC assunta dal paziente. Pertanto, è sempre difficile interpretare e confrontare i risultati ottenuti da diversi laboratori. Inoltre, vi sono implicazioni in termini di costi che devono essere prese in considerazione se vengono periodicamente richieste valutazioni della CPR, considerando la scarsa affidabilità di quest'ultima nell'aiutare in modo significativo a guidare le strategie di sostituzione. La compliance farmacologica è un ulteriore fattore che deve essere considerato $\mathrm{e}$, in molti casi, le dosi prescritte non riflettono necessariamente ciò che viene effettivamente assunto dal paziente.

Ciò è particolarmente vero nei pazienti con ISC, di cui è noto che fino a un terzo degli adulti risulta non aderire alla terapia in maniera corretta. Nella nostra coorte di pazienti adulti con ISC-PS non vi era alcuna relazione tra CPR e il controllo della malattia (in termini di livelli di $17-\mathrm{OH}$ progesterone e androstenedione). Questo suggerirebbe che, in presenza di una buona aderenza alla terapia sostitutiva con GC, valori alterati di CPR potrebbero probabilmente dipendere da una dose sostitutiva di MC insufficiente.

Nel loro insieme, comunque, questi fattori hanno indubbiamente contribuito all' ampia variabilità dei valori di CPR che abbiamo osservato e alla mancanza di relazione con la dose sostitutiva di MC e le variabili cliniche analizzate, confermando le osservazioni fatte finora in precedenti studi con numerosità più contenute.

Il nostro studio ha ovviamente dei limiti. Si tratta di un'analisi retrospettiva condotta in diversi centri, quindi esiste il potenziale di errore derivato da un'elevata eterogeneità della popolazione di studio. Allo stesso modo, la misurazione della renina plasmatica non è stata centralizzata, ma analizzata con diverse metodiche nei centri partecipanti. Inoltre, non è stato possibile determinare l'impatto della terapia sostitutiva con GC, a causa della mancanza di informazioni sulla formulazione e sulla dose utilizzata dai pazienti. I dati inclusi nello studio provengono dalla pratica clinica ambulatoriale e non da una sperimentazione clinica controllata standardizzata. Ciò è particolarmente rilevante nell'interpretazione dell'analisi longitudinale in cui la titolazione della dose MC è stata effettuata in base alle preferenze del medico piuttosto che a un algoritmo specifico stabilito. Sono necessari studi prospettici per ridurre gli effetti dei fattori confondenti attraverso un approccio dedicato e rigoroso per chiarire il contributo di diverse variabili cliniche e biochimiche sulla RPC e, successivamente, sulla titolazione del dosaggio MC. Sebbene il disegno del nostro studio sia chiaramente un limite, questo offre un vero spaccato della pratica clinica routinaria e delle variabili che vengono utilizzate dai clinici al fine di ottimizzare la gestione terapeutica dei pazienti con ISP.

\section{Conclusioni}

In conclusione, il monitoraggio di routine degli elettroliti sierici (insieme alla valutazione clinica dei sintomi e della PA) fornisce l'approccio più informativo da aggiungere alla CPR quando è necessario regolare la sostituzione della MC. Tuttavia, in assenza della capacità di standardizzare accuratamente il dosaggio della CPR, la sola sua misurazione di routine può essere in conflitto con altri strumenti utilizzati per valutare l'adeguatezza della sostituzione della MC. Le decisioni di modificare la dose di MC, quindi, non dovrebbero essere basate esclusivamente sulla CPR.

Sono necessari studi prospettici su larga scala, standardizzati e dedicati a determinare in modo conclusivo il ruolo della PRC nel monitoraggio della sostituzione MC nei pazienti ISP. 
Funding Note Open access funding provided by Università degli Studi di Roma La Sapienza within the CRUI-CARE Agreement.

Conflitto di interesse Gli autori Riccardo Pofi e Elisa Giannetta dichiarano di non avere conflitti di interesse.

Consenso informato Lo studio presentato in questo articolo non ha richiesto sperimentazione umana.

Studi sugli animali Gli autori di questo articolo non hanno eseguito studi sugli animali.

Nota della casa editrice Springer Nature rimane neutrale in riguardo alle rivendicazioni giurisdizionali nelle mappe pubblicate e nelle affiliazioni istituzionali.

Open Access This article is licensed under a Creative Commons Attribution 4.0 International License, which permits use, sharing, adaptation, distribution and reproduction in any medium or format, as long as you give appropriate credit to the original author(s) and the source, provide a link to the Creative Commons licence, and indicate if changes were made. The images or other third party material in this article are included in the article's Creative Commons licence, unless indicated otherwise in a credit line to the material. If material is not included in the article's Creative Commons licence and your intended use is not permitted by statutory regulation or exceeds the permitted use, you will need to obtain permission directly from the copyright holder. To view a copy of this licence, visit http://creativecommons.org/licenses/by/4.0/.

\section{Bibliografia}

1. Speiser PW, Arlt W, Auchus RJ et al (2018) Congenital adrenal hyperplasia due to steroid 21-hydroxylase deficiency: an Endocrine Society clinical practice guideline. J Clin Endocrinol Metab 103(11):4043-4088

2. Oelkers W, Diederich S, Bahr V (1992) Diagnosis and therapy surveillance in Addison's disease: rapid adrenocorticotropin (ACTH) test and measurement of plasma ACTH, renin activity, and aldosterone. J Clin Endocrinol Metab 75(1):259-264

3. Thompson DG, Mason AS, Goodwin FJ (1979) Mineralocorticoid replacement in Addison's disease. Clin Endocrinol (Oxf) 10(5):499-506

4. Pofi R, Prete A, Thornton-Jones V et al (2020) Plasma renin measurements are unrelated to mineralocorticoid replacement dose in patients with primary adrenal insufficiency. J Clin Endocrinol Metab 105(1):dgz055 